\title{
Mean Value Theorem for Functions Possessing First Order Convex Approximations. Applications in Optimization. Theory
}

\author{
M. StUdNIARski
}

In dieser Arbeit wird ein Mittelwertsatz für Funktionen abgeleitet, die konvexe Approximationen erster Stufe im Sinne von IOFFE [6] besitzen. Er umfaßt zwei bekannte Ergebnisse für konvexe und lokal Lipschitzsche Funktionen als Spezialfälle. Dieser Satz wird zur Ableitung einer hinreichenden Bedingung dafür verwendet, da $B$ eine Funktion, die auf kartesischem Produkt zweier topologischer Vektorräurne definiert ist, eine konvexe Approximation erster Stufe besitzt. Einige Anwendungen in der Optimierungstheorie werden auch dargestellt.

В работе доказана теорема о среднем для функций, облацающих выпуклыми аппроксимациями первого порядка в смысле Иоффе [6]. Она включает в себя два известных результата для выпуклых и локально липшицевых функция как частные случаи. Эта теорема применяется для вывода достаточного условия для того, чтобы функция, определенная на прямом произведении двух топологических векторных пространств, обладала выпуклой аппроксимациеи первого порядка. Представлены тоже некоторые приложения әтих результатов в теории оптимизации.

A mean value theorem fór functions possessing first order convex approximations in the sense of Ioffe [6] is derived. It comprises two known results for convex and locally Lipschitzian functions as particular cases. This theorem is used in order to obtain a sufficient condition for a function defined on the Cartesian product of two topological vector spaces to possess, a first order convex approximation. Some applications in optimization theory are also given.

\section{Introduction}

In the last few years there appeared many publications in which mean value theorems for various classes of nondifferentiable real- and vector-valued functions were derived (see $[3,4,7,9,10,12]$ for the most general results). These theorems were stated by means of various generalizations of the notion of a gradient (or derivative) in differential calculus and of a subdifferential in convex analysis. In this paper we present a mean value theorem for real-valued functions (on arbitrary real topological vector spaces) possessing first order convex approximations in the sense of IoFFE [6]. This kind of approximation plays an important role in the theory of first order necessary optimality conditions (it requires weaker assumptions than other approximations used in many earlier papers). A first order convex approximation of a function is not 'uniquely determined and, by taking its subdifferential, one can obtain different generalizations of the derivative of the function being approximated.

As corollaries from our mean value theorem (Theorem 1), we obtain the results of Hiriart-URRoty [3] for convex lower semicontinuous and of LEBoURG [9] for locally Lipschitzian functions. From Theorem 1 as well, we derive a sufficient condition (Theorem 2) for the existence of a first order convex approximation for a function defined on the Cartesian product of two topological vector spaces. Then we apply Theorem 2 to obtain a variant of the extremum principle of Iofre and Tukno- 
MmRov [8], and Theorem 1 to generalize the sufficient optimality condition of DYCKHOFF [2].

Throughout the paper, the letters $X, Y$ will denote (unless otherwise stated) real topological vector spaces. Let $f: X \rightarrow \mathbf{R} \cup\{+\infty\}$ be convex and proper (i.e. $\operatorname{dom} f$ $=\{x \in X \mid f(x)<\infty\} \neq \varnothing)$. We shall denote by $\partial f(x)$ the subdifferential of $f$ at the point $x$, that is,

$$
\partial f(x)=\left\{x^{*} \in X^{*}, \forall u \in X, f(u) \geqq f(x)+\left\langle x^{*}, u-x\right\rangle\right\}
$$

where $X^{*}$ is the topological dual space of $X$. It can be shown (cf. [8: $\S 4.2$, Prop. 1]) that, for each $x \in \operatorname{dom} f$,

$$
\partial f(x)=\left\{x^{*} \in X^{*} \mid \forall h \in X, f^{\prime}(x ; h) \geqq\left\langle x^{*}, h\right\rangle\right\}
$$

where

$$
f^{\prime}(x ; h)=\lim _{\lambda \downarrow 0^{-}} \lambda^{-1}(f(x+\lambda h)-f(x)) .
$$

For two different points $a$ and $b$ in $X$, we shall denote, respectively, by $[a, b]$ and ]$a, b[$ the subsets

$$
\{a+t(b-a) \mid 0 \leqq t \leqq 1\} \text { and }\{a+t(b-a) \mid 0<t<1\} .
$$

\section{First order convex approximations and Dini derivatives}

Let $f: U \rightarrow \mathbf{R}$ be a real-valued function defined on an open subset $U$ of $X$, and let $x \in U$. We depote, for any $h \in X$,

$$
f_{+}^{\prime}(x ; h)=\lim _{\lambda \downarrow 0} \sup \lambda^{-1}(f(x+\lambda h)-f(x)) \text {. }
$$

A function $\varphi: X \rightarrow \mathbf{R}$ is called a first order convex approximation for $f$ at $x$ if $\varphi$ is sublinear (i.e. convex and $\varphi(\lambda h)=\lambda . \varphi(h)$ for all $\lambda \geqq 0, h \in X)$, continuous and satisfies the condition

$$
f_{+}^{\prime}(x ; h) \leqq \varphi(h) \text { for all } h \in X:
$$

Remarks: 1. The original definition of IOFFE [6] contains instead of (4) the equivalent condition

$$
\limsup _{\lambda \downarrow 0} \lambda^{-1}(f(x+\lambda h)-f(x)-\lambda \varphi(h)) \leqq 0 .
$$

The convexity and continuity assumptions are not included in this definition, but used in all subsequent theorems.

2. Instead of the continuity of $\varphi$, it suffices to assume that $\varphi$ is bounded above on some open subset of $X$ (then $\varphi$ is continuous by [8: $\S 3.2$, Theorem 1]). Since $\varphi$ is convex and continuous, the subdifferential $\partial \varphi(0)$ is non-empty (the proof of this fact without the assumption of the local convexity of $X$ is given in [5: $\S 14 \mathrm{~B}]$ ). By (1) we have

$$
\partial \varphi(0)=\left\{x^{*} \in \dot{X}^{*} \mid \forall h \in X, \varphi(h) \geqq\left\langle x^{*}, h\right\rangle\right\} .
$$

Moreover, by [6: Prop. 1,

if $f$ has a local minimum at $x$, then $0 \in \partial \varphi(0)$. 
Examples: 1. Let $U$ be convex and $f-$ convex and continuous. Then, for each $x \in U$ and $h \in X$, the directional derivative (3) exists and is finite, and the function $f^{\prime}(x ; \cdot): X \rightarrow \mathbf{R}$ is sublinear and continuous (cf. [8: $\S 4.1$, Prop. 4]). Therefore $f^{\prime}(x ; \cdot)$ is a first.order convex approximation for $f$ at $x$.

2. Let $f$ bé locally Lipschitzian in the sense of [9], that is, the generalized directional derivative

$$
f^{\circ}(x ; h) \doteq \limsup _{\substack{u \rightarrow x \\ \lambda \downarrow 0}} \lambda^{-1}(f(u+\lambda h)-f(u))
$$

satisfies the following condition: for each $a \in U$, there exist a neighbourhood $A$ of $a$ in $U$ and a circled neighbourhood $W$ of 0 in $X$, such that the set $\left\{f^{\circ}(x ; h) \mid x \in A\right.$, $h \in W\}$ is bounded in R. It follows from [9: Lemria (1.1) and Prop. (1.2)] that $f^{\circ}(x ; \cdot)$ is a first order convex approximation for $f$ at $x$. If $f$ is as described in Example 1, then it is locally Lipschitzian and $f^{\circ}=f^{\prime}$ by [9: Prop. (1.10)].

Let us now consider a function $f: V \rightarrow \mathbf{R}$ where $V$ is an open subset of $\mathbf{R}$. We define the following Dini derivatives of $f$ at $x \in V$

$$
\begin{aligned}
& D^{+} f(x)=\limsup _{\lambda \downarrow 0} \lambda^{-1}(f(x+\lambda)-f(x)), \\
& D_{+} f(x)=\underset{\lambda \downarrow 0_{1}}{\lim \lambda_{i n f}} \lambda^{-1}(f(x+\lambda)-f(x)) .
\end{aligned}
$$

and, analogously, $D^{-} f(x)$ and $D_{-} f(x)$ by taking the limits as $\lambda \uparrow 0$.

Proposition 1: A function $\varphi: \mathbf{R} \rightarrow \mathbf{R}$ is a first order convex approximation for $f$ at $x$ if and only if $\varphi$ is sublinear and $-D_{-} f(x) \leqq \varphi(-1), D^{+} f(x) \leqq \varphi(1)$.

Proof: It suffices to observe that $-D_{-} f(x)=f_{+}^{\prime}(x ;-1), D^{+} f(x)=f_{+}^{\prime}(x ; 1)$

Proposition 2: Let $f:[a, b] \rightarrow \mathbf{R}($ where $a<b)$ be continuous ańd satisfy the conditions:

a) for each $x \in] a, b\left[\right.$, there exists, a first order convex' approximation $\varphi_{x}$ for f at $x$;

b) the functions $x \mapsto \varphi_{x}(-1)$ and $x \mapsto \varphi_{x}(1)$ are upper semicontinuous on $] a, b[$.

If $f$ attains a local maximum at $c \in] a, b\left[\right.$, then $0 \in \partial \varphi_{c}(0)$.

Proof: Suppose that $f$ has a local maximum at $c$, and $0 \notin \partial \varphi_{c}(0)$. Hence (by (5)) either $\varphi_{c}(1)<0$ or $\varphi_{c}(-1)<0$. In the first case it follows from assumption b) and Proposition 1 that there exists $\varepsilon>0$ such that

$$
\left.D^{+} f(x) \leqq \varphi_{x}(1)<0 \text { for all } x \in[c-\varepsilon, c] \subset\right] a, b[\text {. }
$$

For such $x$, we have $D^{+}(-f)(x)=-D_{+} f(x) \geqq-D^{+} f(x)>0$. Hence and from [1: Cor. XI.4.2] we obtain that $-f$ is non-decreasing on $[c-\varepsilon, c]$, thus $f(x) \geqq f(c)$ for all $x \in[c-\varepsilon, c]$. Since $f$ has a local maximum at $c$, there exists $\delta \in] 0, \varepsilon[$ such that $f(x) \leqq f(c)$ for all $x \in[c-\delta, c]$. Therefore $f$ is constant on $[c-\delta, c]$ and $D^{+} f(c-\delta)$ $=0$, which contradicts (7). The case of $\varphi_{c}(-1)<0$ can be considered analogously (instead of $-f$ one should take into account the function $g(x)=-f(-x)$ and observe that $D_{*}^{+} g(-x) \geqq D_{+} g(-x)=D_{-} f(x)>0$ for all $x$ in' some interval $\left.\left[c, c+\varepsilon^{\prime}\right], \varepsilon^{\prime}>0\right)$

Remark: Proposition 2 is not true without assumption b). As a counterexample it suffices to take $f(x)=-|x|, \varphi_{x}(u)=u$ for $x<0, \varphi_{x}(u)=-u$ for $x \geqq 0, c=0$. 


\section{The mean value theorem}

Let $A$ be a subset of $X$ with nonempty interior, and let $[a, b] \subset A$. We shall say that a function $f: A \rightarrow \mathbf{R}$ is hemi-continuous on $[a, b]$ (cf. [4]) if the function $i \mapsto f(a$ $+t(b-a))$ is continuous on $[0,1]$.

Theorem 1: Let $f: A \rightarrow \mathbf{R}$ satisfy the conditions:

a) $f$ is hemi-continuous on $[a, b]$;

b) ] $a, b[\subset$ int $A$ and, for each $x \in] a, b\left[, \varphi_{x}\right.$ is a first order convex approximation, for $f$ at $x$

c) the functions $x \mapsto \dot{\varphi}_{x}(a-b)$ and $x \mapsto \dot{\varphi}_{x}(b-a)$, defined on ]a, $b[$, are upper semicontinuous.

Then

there exist $c \in] a, b\left[\right.$ and $x^{*} \in \partial \varphi_{c}(0)$ such that

$$
f(b)-f(a)=\left\langle x^{*}, b-a\right\rangle \text {. }
$$

Proof: Let us first consider the case when $X=\dot{\mathbf{R}}$ (and $a<b$ ). If $f(a)=f(b)$, then there exists $c \in] a, b$ [ which is a local minimum or maximum point for $f$. Hence $0 \in \partial \varphi_{c}(0)$ by (6) or Proposition 2, and (8) holds for $x^{*}=0$. If $f(a) \neq f(b)$, then instead of $f$ one should consider the funtion $f(x)=f(x)-f(a)-\alpha(x-a)(x \in A)$ where $\alpha$ $=(f(b)-f(a)) \mid(b-a)$. It is easy to verify that $\bar{\varphi}_{x}(h)=\varphi_{x}(h)-\alpha h(h \in \mathbf{R})$ is a first order convex approximation for $\bar{f}$ at $x \in] a, b\left[\right.$, and that $\partial \bar{\varphi}_{c}(0)=\partial \varphi_{c}(0)-\alpha$; hence (8) holde for $x^{*}=\alpha$.

In the general case we put $g(t)=f(a+t(b-a)), t \in[0,1]$, and observe that, for each $t \in] 0,1\left[\right.$, the function $\psi_{t}(s)=\varphi_{a+t(b-a)}(s(b-a))(s \in \mathbf{R})$ is a first order convex approximation for $g$ at $t$, and that the functions $t \mapsto \psi_{t}(-1)$ and $t \mapsto \psi_{t}(1)$ are upper semicontinuous on ]0, 1 [. Applying the result just proved to the function $g$, we obtain that there exist $r \in.] 0,1\left[\right.$ and $\beta^{\prime} \in \partial \psi,(0)$ such that

$$
\beta=g(1)-g(0)=f(b)-f(a) \text {. }
$$

Let $c=a+r(b-a)$. Since $\beta \in \partial \psi_{r}(0)$, we have $\beta s \leqq \varphi_{c}(s(b-a))$ for all $s \in \mathbf{R}$. We define a continuous linear functional $l$ on the subspace $\{x \in X \mid x=s(b-a), s \in \mathbf{R}\}$ by setting $l(s(b-a))=\beta s$. It follows from the Hahn-Banach theorem [5: $11 \mathrm{G}]$ that $l$ has an extension $x^{*} \in X^{*}$ satisfying $\left\langle\dot{x}^{*}, h\right\rangle \leqq \varphi_{c}(h)$ for all $h \in X$. Since $\left\langle x^{*}\right.$, $b-a\rangle=l(b-a)=\beta$, we obtain (8) by (5) and (9)

Corollary 1 [3: Theorem 7]: Let $f: X \rightarrow \mathbf{R} \cup\{+\infty\}$ be convex and lower semicontinuous. Suppose that $[a, b] \subset \operatorname{dom} f$, and that $f$ is continuous at some point of $[a, b]$. Then

there exist $c \in] a, b\left[\right.$ and $x^{*} \in \partial f(c)$ such that

$$
f(b)-f(a)=\left\langle x^{*}, b-a\right\rangle \text {. }
$$

Proof: Since $f$ is continuous at a point of $[a, b]$, we have $] a, b[\subset$ int (dom $f$ ), and $f$ is continuous on int (dom $f)^{\text {by }}$ [8: $\$ 3.2$, Theorem 1]: It is easy to show (by making use of Example 1) that $f$ restricted to $A=\operatorname{dom} f$ satisfies the assumptions of Theorem 1 (the upper semicontinuity of $f^{\prime}(\cdot ; h)$ on int $A$ can be deduced from the continuity of $f$ and the equality $f^{\prime}(x ; h)=\inf \left\{\lambda^{-1}(f(x+\lambda h)-f(x)) \mid \lambda>0\right\}$, similarly as in the proof of Corollary 3 below). Thus (10) follows from (8)

The following result is a direct consequence of Theorem 1, Example 2 and the upper semicontinuity of $f^{\circ}(\cdot ; h)$. 
Corollary 2 [9: Theorem (1.7)]: If $A$ is open and $f: A \rightarrow \mathbf{R}$ is locally Lipschitzian, then $(10)$ is true, where $\partial f(c)$ denotes the generalized gradient of $f$ at $c$ (i.e. the subdifferential of $f^{\circ}(c ; \cdot)$ at 0$)$.

\section{Applications}

Let us consider the following problem: given a function $f: X \times Y \rightarrow, \mathbf{R}$ such that the functions $f(x, \cdot)$ and $f(\cdot, y)$ possess first order convex approximations, we want to formulate sufficient conditions (stated by means of these "partial" approximations) for the existence of an approximation for $f$ at $(x, y)$ in $X \times Y$.

Theorem 2: Let $U$ and $V$ be open subsets of $X$ and $Y$, respectively, and let $(x, y)$ $\epsilon U \times V$. Suppose that $f: U \times V \rightarrow \mathbf{R}$ satisfies the conditions:

a) for any $u \in U$, the function $f(u, \cdot): \nabla \rightarrow \mathbf{R}$ is hemi-continuous on each segment $\left[y, y^{\prime}\right] \subset \nabla$ and has at each point $v \in V$ a first order convex approximation $\psi_{u, v}: Y \rightarrow \mathbf{R}$;

b) the function $f(\cdot, y): U \rightarrow \mathbf{R}$ has a first order convex approximation $\varphi: X \rightarrow \mathbf{R}$.at the point $x$;

c) for each $k \in Y$, the function $(u, v) \mapsto \psi_{u, v}(k)$ is upper semicontinuous on $U \times V$.

Then the function $\omega: X \times Y \rightarrow \mathbf{R}$ defined by

$$
\omega(h, k) \stackrel{=}{=}(h)+\psi_{x, y}(k) \quad(h \in \dot{X}, k \in Y)
$$

is a first order convex approximation for $f$ at ${ }^{-}(x, y)$.

Proof: The only difficulty is to check condition (4) of the definition, which is equivalent to the following one:

$$
\begin{aligned}
& \text { for each } \varepsilon>0 \text { and }(h, k) \in X \times Y \text {, there exists } \mu>0 \text { such that } \\
& \left.\lambda^{-1}(f(x+\lambda h, y+\lambda k))-f(x, y) \leqq \omega(h, k)+{ }^{-} \varepsilon \text { for all } \lambda \in\right] 0, \mu[\text {. }
\end{aligned}
$$

Take any $\varepsilon>0, h \in X, k \in Y$. By c), there exist neighbourhoods $U^{\prime} \subset \dot{U}$ and $V^{\prime} \subset V$ of $x$ and $y$, respectively, such that

$$
\psi_{u, v}(k) \leqq \psi_{x, y}(k)+\varepsilon / 2 \text { for all } u \in \dot{U}^{\prime}, v \in \nabla^{\prime} .
$$

By b), there exists $\mu>0$ such that

$$
\begin{aligned}
& x+\lambda h \in U^{\prime}, \quad y+\lambda k \in \nabla^{\prime} \text { and } \\
& \left.\hat{\lambda}^{-1}(f(x+\lambda h ; y)-f(x, y)) \leqq \varphi(h)^{\prime}+\varepsilon / 2, \quad \text { for all } \quad \lambda \in\right] 0, \mu[.
\end{aligned}
$$

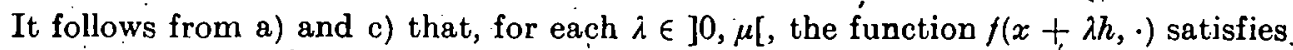
the assumptions of Theorem 1 on the segment $[y, y+\lambda k]$. Consequently, there exist $\left.c_{\lambda} \in\right] y, y+\lambda k\left[\right.$ and $x_{\lambda}^{*} \in \partial \psi_{x+\lambda h_{,} c_{\lambda}}(0)$, such that $f(x+\lambda h, y+\lambda k)-f(x+\lambda h, y)$ $=\left\langle x_{\lambda}^{*}, \lambda, k\right\rangle$. Hence and from (12), (13) and (5) we obtain

$$
\begin{aligned}
& \lambda^{-1}(f(x+\lambda h, y+\lambda k)-f(x, y)) \\
& =\lambda^{-1}(f(x+\lambda h, y+\lambda k)-f(x+\lambda h, y))+\lambda^{-1}(f(x+\lambda h, y)-f(x, y)) \\
& \leqq\left\langle x_{\lambda}{ }^{*}, k\right\rangle+\varphi(h)+\varepsilon / 2 \leqq \varphi(h)+\psi_{x+\lambda, c_{\lambda}}(k)+\varepsilon / 2 \leqq \varphi(h)+\psi_{x, y}(k)+\varepsilon,
\end{aligned}
$$

which concludes the proof of (11)

Corollary 3: Let $f: U \times \nabla \rightarrow \mathbf{R}$ be a continuous function, where $U$ and $V$ are open subsets of $X$ and $Y$, respectively, and $V$ is convex. Let $(x, y) \in U \times \nabla$. If, for each 
$u \in U, f(u, \cdot): V \rightarrow \mathbf{R}$ is convex and assumption $\mathrm{b})$ of Theorem $\mathbf{2}$ is fulfilled, then $(h, k) \mapsto \varphi(h)+f_{y}^{\prime}(x, y ; k)$ is a first order convex approximation for $f$ at $(x, y)$ (here $f_{y}{ }^{\prime}(x, y ; k)$ denotes the directional derivative of $f(x, \cdot)$ at $y$ in the direction $\left.k\right)$.

Proof: It follows from Example 1 that assumption a) of Theorem 2 is satisfied for $\psi_{u, v}=f_{y}{ }^{\prime}(u, v ; \cdot)$. Thus it suffices to prove that, for each $k \in Y$, the function $(u, v)$ $\mapsto f_{y}^{\prime}(u, v ; k)$ is upper semicontinuous on $U \times \bar{V}$. Take any $\varepsilon>0, \bar{u} \in U, \bar{v} \in V$. Since

$$
f_{y}^{\prime}(\bar{u}, \bar{v} ; k)=\inf \left\{\lambda^{-1}(f(\bar{u}, \bar{v}+\lambda k)-f(\bar{u}, \bar{v})) \mid \lambda>0\right\}
$$

(cf. [8: $\$ 4.1]$ ), there exists $\mu>0$ such that $\bar{v}+\mu k \in V$ and .

$$
\mu^{-1}(f(\bar{u}, \bar{v}+\mu k)-f(\bar{u}, \bar{v})) \leqq f_{\nu}{ }^{\prime}(\bar{u}, \bar{v} ; k)+\varepsilon / 2 .
$$

By the continuity of $f$ at the points $(\bar{u}, \bar{v})$ and $(\bar{u}, \bar{v}+\mu k)$, there exist neighbourhoods $U^{\prime} \subset U$ and $V^{\prime} \subset V$ of $\bar{u}$ and $\bar{v}$, respectively, such that

$$
\max (|f(u, v)-f(\bar{u}, \bar{v})|,|f(u, v+\mu k)-f(\bar{u}, \bar{v}+\mu k)|) \leqq \varepsilon \mu / 4
$$

for all $u \in U^{\prime}, v \in V^{\prime}$. Hence and from (14) we obtain that, for all $u \in U^{\prime}$ and $v \in V^{\prime}$,

$$
\begin{aligned}
& f_{v}^{\prime}(u, v ; k)=\inf \left\{\lambda^{-1}(f(u, v+\lambda k)-f(u, v)) \mid \lambda>0\right\} \\
& \leqq \mu^{-1}(f(u, v+\mu k)-f(u, v)) \\
& =\mu^{-1}(f(u, v+\mu k)-f(\dot{u}, \bar{v}+\mu k))+\mu^{-1}(f(\bar{u}, \bar{v}+\mu k)-f(\bar{u}, \bar{v})) \\
& \quad+\mu^{-1}(f(\bar{u}, \bar{v})-f(u, v)) \leqq f_{\nu}{ }^{\prime}(\bar{u}, \bar{v} ; k)+\varepsilon,
\end{aligned}
$$

and so $(u, v) \mapsto f_{y}^{\prime}(u, v ; k)$ is upper semicontinuous at $(\bar{u}, \bar{v})$

We shall now apply the above corollary to obtain a variant of the Ioffe-Tikhomirov extremum principle (cf. [8: $\S \S^{\prime} 1.1$ and 5.1]). Let us consider the following extremal problem

$$
\begin{aligned}
& \text { minimize } f_{0}(x, y) \text { subject to } \\
& f_{i}(x, y) \leqq 0(i=1, \ldots, n), . F(x, y)=0, \quad y \in U^{\prime}
\end{aligned}
$$

where $U \subset Y, f_{i}: X \times Y \rightarrow \mathbf{R}(i=0,1, \ldots, n), F: X \times Y \rightarrow Z(X, Y, Z$ - normed spaces).

, Theorem 3: Let $(\bar{x}, \bar{y}) \in X \times U$. Assume that

a) $f_{i}(i=0,1, \ldots, n)$ and $F$. satisfy the Lipschitz condition on a neighbourhood of $i(\bar{x}, \bar{y})$;

b) $U$ is convex and closed;

c) there are first order convex approximations $\varphi_{i}(i=0,1, \ldots, n)$ and $\psi$ for $f_{i}(\cdot, \bar{y})$ and $\|F(\cdot, \bar{y})\|$, respectively, at $\bar{x}$

d) for each $x \in X, f_{i}(x, \cdot)(i=0,1, \ldots, n)$ and $\|F(x, \cdot)\|$ are convex on $Y$;

e) $(\bar{x}, \bar{y})$ is a régular point for $F$ relative to $X \times U$ (i.e. there are $\alpha>0$ and a neighbourhood $\nabla$ of $(\bar{x}, \bar{y})$, such that, for all $(x, y) \in \nabla \cdot \cap(X \times U), d_{Q}(x, y) \leqq \alpha \| F(x, y)$ - $F(\bar{x}, \bar{y}) \|$ where $Q=\{(x, y) \in X \times U \mid F(x, y)=F(\bar{x}, \bar{y})\}$ and $d_{Q}$ denotes the distance function to $Q)$.

If $(\bar{x}, \bar{y})$ is a local solution to (15), then there exist numbers

$$
\lambda_{i} \geqq 0 \cdots:(i=0,1, \ldots, n), \quad r>0,
$$


such that

$$
\begin{aligned}
& \lambda_{0}+\cdots+\lambda_{n}=1, \quad \lambda_{i} f_{i}(\bar{x}, \bar{y})=0 \text { for } i=1, \ldots, n, \\
& 0 \in \sum_{i=0}^{n} \lambda_{i} \partial \phi_{i}(0)+r \partial \psi(0), \\
& \sum_{i=0}^{n} \lambda_{i} f_{i}(\bar{x}, \bar{y})+r\|F(\bar{x}, \bar{y})\| \\
& =\min _{y \in U}\left(\sum_{i=0}^{n} \cdot \lambda_{i} f_{i}(\bar{x}, y)+\cdot r\|F(\bar{x}, y)\|\right) .
\end{aligned}
$$

Proof: Put $g(x, y)=\|F(x, y)\|$. From Corollary 3 we get that the functions $\omega_{i}(h, k)$ $=\varphi_{i}(h)+\left(f_{i}\right)_{y}{ }^{\prime}(\bar{x}, \bar{y} ; k)(i=0,1, \ldots, n), \quad \eta(h, k)=\psi(h)+g_{y}{ }^{\prime}(\dot{x}, \bar{y} ; k)$ and $\xi(h, k)$ $=d_{\sigma^{\prime}}(\bar{y} ; k)$. are first order convex approximations for $f_{i},\|F\|$ and $d_{X \times U}$, respectively, at. $(\bar{x}, \bar{y})$. Hence and from [6: Theorem 2] we obtain that there exist numbers (16) satisfying conditions (17) and

$$
\text { , } 0 \in \sum_{i=0}^{n} \lambda_{i} \partial \omega_{i}(\theta, 0)+r \partial \eta(0,0)+r_{i} \partial \xi(0,0) \text {. }
$$

It is easy to verify (cf. [11: Lemma 2]) that, for any continuous convex function $\omega$ : $X \times Y \rightarrow \mathbf{R}$, if $z^{*} \in \partial \omega(x, y)$, then there exist $x^{*} \in \partial(\omega(\cdot, y))(x)$ and $y^{*} \in \partial(\omega(x, \cdot))(y)$ such that $\left\langle z^{*}, .(u, v)\right\rangle^{\prime}=\left\langle x^{*}, u\right\rangle+\left\langle y^{*}, v\right\rangle$ for all $u \in X, v \in Y$. Thus, from (20) we obtain (18) and (by (2)) ".

$$
0 \epsilon \sum_{i=0}^{n} \lambda_{i} \partial^{\prime}\left(f_{i}(\bar{x}, \cdot)\right)(\bar{y})+r \partial(g(\bar{x}, \cdot))(\bar{y})+r \ddot{\partial} d_{U}(\bar{y}) \text {. }
$$

We readily deduce from the definition of the subdifferential (formula (1)) that

$$
r \partial d_{U}(\bar{y}) \subset \partial(\delta(\cdot \mid U))(\bar{y})
$$

where $\delta(\cdot \mid U)$ is defined as follows:

$$
\delta(v \mid \dot{U})= \begin{cases}0 & \text { if } v \in U, \\ +\infty & \text { if } v \notin U .\end{cases}
$$

Consequently, by the Moreau-Rockafellar theorem [8: $§ 0.3],(21)$ and (22) imply

$$
0 \in \partial\left(\sum_{i=0}^{n} \lambda_{i} f_{i}(\bar{x}, \cdot)+r\|F(\bar{x}, \cdot)\|+\delta(\cdot \mid U)\right)(\bar{y}),
$$

which is equivalent to (19)

We shall now give another application of Theorem 1 : to éxtend the sufficient opti-. mality condition of DYCKHOFF [2] to the case of functions not necessarily directionally differentiable. Let $U$ be a convex subset of $X$ with nomempty interior, and let $\bar{x} \in U$. Suppose that a function $f: U \rightarrow \mathbf{R}$ has first order convex approximations at all points of the set $\tilde{U}=$ (int $U$ ) \\{ $\bar{x}\}$, but may have no approximation at $\bar{x}$. We shall formulate a sufficient condition for $\bar{x}$ to be the global minimum point of $f$ on $U$.

Proposition 3: Assume that

a) $f$ is hemi-continuous on each segment $[a, b] \subset U$;

b) f has a first order convex approximation $\varphi_{x}$ at each $x \in \tilde{U}$; 
c) for each $y \in \tilde{U}$, the functions $x \mapsto \varphi_{x}(\bar{x}-y)$ and $x \mapsto \varphi_{2}(y-\bar{x})$, restricted to ] $\bar{x}, y[$, are upper semicontinuous;

d) $\varphi_{y}(\bar{x}-y)^{\prime} \leqq 0$ for all $y \in \tilde{U}$.

Then $f(\bar{x}) \leqq f(x)$ for all $x \in U$.

Proof: Fix $x \in \tilde{U}$. By Theorem 1, there exist $c \in] \bar{x}, x\left[\right.$ and $x^{*} \in \partial \varphi_{c}(0)$, such that $f(\bar{x})-f(x)=\left\langle x^{*}, \bar{x}-x\right\rangle \leqq \varphi_{c}(\bar{x}-x)$. Since $\dot{c} \in \tilde{U}$, we have (by'd $\left.)\right) \varphi_{c}(\bar{x}-c) \leqq 0$. But $\bar{x}-c=\lambda(\bar{x}-x)$ for some $\lambda>0$; hence $\varphi_{c}(\bar{x}-x) \leqq 0$ and $f(\bar{x}) \leqq f(x)$.

Assume now that $x \in U \backslash \tilde{U}$. Take any $u \in \tilde{U}$. It follows from a) and from the convexity of $U$.that, for each $\varepsilon>0$, there exists $w \in] x, u[n \tilde{U}$ such that $f(w) \leqq f(\dot{x})+\varepsilon$. By the first part of the proof, $f(\bar{x}) \leqq f(w)$. Thus $f(\bar{x}) \leqq f(x)$

\section{REFERENCES}

[1] Brdckner, A. M.: Differentiation of real functions (Lecture Notes in Mathematics: Bd. 65்9). Berlin-Heidelberg-New York: Springer-Verlag 1978.

[2] Dyскноғf, H.: An optimality criterion for problems without differentiability on the boundary. Z. Oper. Res. Ser. A 25 (1981), 167-169.

[3] Hiriart-Urroty, J.-B.: A note on the mean value theorem for convex functions. Boll. Un. Mat. Ital. B (5) 17 (1980), 765-775.

[4]. Hiniart-Urrdty, J.-B.: Mean value theorems in nonsmooth analysis. Numer. Funct. Anal. Optim. 2 (1980), $1-30$.

[5] Holmes, R. B.: Geometric functional analysis and its applications. Berlin-HeidelbergNew York: Springer-Verlag 1975.

[6] Ioffe, A. D.: Necessary and sufficient conditions for a local minimum. 1: A reduction theorem and first order conditions. SIAM J. Control Optim. 17 (1979), 245-250.

[7.] Iófre, A. D.: Nonsmooth analysis: differential calculuś of nondifferentiäble mappings. Trans. Amer. Math. Soc. 266 (1981), $1-56$.

[8] Иоффе, А. Д., и В. М. Тихомичов: Теория әкстремальных задач. Москва: Изд-во Наука 1974.

[9] Lebourg, G.: Generic differentiábility of Lipschitzian functions. Trans. Amer. Math. Soc. 256 (1979), $125-144$.

[10].Poorcis U, B. H: : Analysis and optimization of Lipschitz continuous mappings: J. Optim. Theory Appl. 22 (1977), 311-351.

[11] StudnrARski, M.: Application of the Dubovitskii-Milyutin method to some locally convex extremal problems. Bull. Soc. Sci. Lett. Łódź 29 (1979) 6, 1-8.

[12] THваULT, I.: On generalized differentials and subdifferentials of Lipschitz vectorvalued functions. Nonlinear Anal. 6 (1982), 1037-1053.

Manuskripteingang: 31.10 .1983

\section{VERFASSER:}

Dr. Marcin Studniarski

Institute of Mathematics, University of Lódź

ॠP-90-238 Łódź, ul. S. Banacha 22 